\title{
Parentalidade Positiva e Bem-Estar Subjetivo: Intervenção com Pais de
}

\section{Estudantes Sobredotados}

\section{Positive Parenting and Subjective Well-Being: Intervention with Parents of Gifted Students}

\author{
Suzy Pinho-Pereira*, Carla Blum Vestena**, Cristina Costa-Lobo* \\ *Universidade Portucalense Infante D. Henrique, ** Durham University
}

\begin{abstract}
Resumo
O apoio sócio-educativo dirigido a pais bem como a intervenção psicopedagógica, fazem parte das políticas sociais europeias, e têm como objetivo nuclear responder às necessidades de educação, promoção do Bem-Estar e desenvolvimento das crianças e jovens. O Programa Parentalidade Positiva é de autoria e é realizado pela Associação Nacional de Estudo e Intervenção em Sobredotação, associação portuguesa. A principal questão explorada nesta investigação: A promoção da parentalidade positiva em pais de crianças sobredotadas tem um impacto positivo no Bem-Estar Subjetivo dos pais? A amostra é não probabilística, de conveniência, contando com um grupo intervenção e um grupo de controlo. Recorre-se a informações demográficas e a um instrumento aferido para a população adulta portuguesa. Esta investigação, assente num estudo quasi experimental, sendo o objetivo principal avaliar $o$ impacto do Programa de Parentalidade Positiva.

Palavras-Chave: Parentalidade Positiva, Bem-Estar Subjetivo, Sobredotação
\end{abstract}

\begin{abstract}
The socio-educational support for parents as well as psychopedagogical intervention are part of European social policies, and have the nuclear objective of responding to the needs of education, the promotion of well-being and the development of children and young people. The Positive Parenting Program is authored and is carried out by the National Association of Study and Intervention in Giving, Portuguese Association. The main question explored in this research: Does promoting positive parenting in parents of gifted children have a positive impact on the subjective well-being of parents? The sample is non-probabilistic, of convenience, counting on an intervention group and a control group. Demographic information is used and a tool checked for the Portuguese adult population. This research is based on a quasi-experimental study, being the main objective evaluate the impact of the Positive Parenting Program.

Keywords: Positive Parenting, Subjective Well-Being, Giftedness
\end{abstract}

\section{Parentalidade Positiva}

Devido aos imensos conceitos que existem na literatura, ainda não é possível definir parentalidade de forma consensual, este termo teve origem nos conceitos de maternidade/ paternidade sendo estas noções distintas. Como o termo de parentalidade, também o termo "Parentalidade Positiva" ainda não tem uma definição global, tendo cada autor a sua própria definição. A Parentalidade só começou a ser um objeto de estudo da Psicologia, das Ciências da Saúde e da Sociologia na segunda metade do século XX (Martins, 2013). O termo parentalidade, originário do latim parentâle, surgiu em meados do ano 1961, após o psicanalista Paul-Claude Racamier propor um significado mais amplo para o termo maternidade (Corvo, 2015). O conceito de parentalidade positiva é definido como o desenvolvimento de padrões de relacionamento saudáveis com os filhos, sendo o comportamento parental baseado no melhor interesse da criança onde assegura a sua educação (Lopes, Catarino, $\&$ Dixe, 2010).

O conceito Parentalidade Positiva procura criar as condições necessárias para que as crianças possam desenvolver as suas capacidades emocionais, sociais, comunicativas, autonomia e de resolução de problemas, de forma mais completa possível dentro ou fora da família, (Baptista, 2014). A Parentalidade Positiva tem como princípios fundamentais o reconhecimento das crianças e dos pais como detentores de direitos e também obrigações, o relacionamento com a criança, o carinho e envolvimento parental positivo são as variáveis essenciais para a prática da parentalidade positiva (Lopes, Catarino \& Dixe, 2010). De acordo com Patrício (2011) a prática da parentalidade positiva envolve mudança, ajustamento e aprendizagem de competências necessárias à tomada de decisão parental, perante a perceção e gestão do comportamento das crianças permitindo aos pais uma progressiva confiança na sua própria parentalidade. É através do relacionamento interpessoal e na comunicação familiar entre pais e filhos que se baseia grande parte das caraterísticas da parentalidade positiva, pois é desenvolvida num estilo parental que abarca uma relação de proximidade e respeito pela criança (Lopes, Catarino \& Dixe, 2010). 


\section{Parentalidade na Sobredotação}

De acordo com as Recomendações do Conselho da Europa, o papel parental positivo é definido como “o conjunto de comportamentos parentais que procura o Bem-Estar das crianças e o seu desenvolvimento integral desde uma perspetiva de cuidado, afeto, proteção, enriquecimento e segurança pessoal, de não violência, que proporciona reconhecimento pessoal e pautas educativas, e inclui o estabelecimento de limites para promover o seu completo desenvolvimento, o sentimento de controlo da sua própria vida" (Gonzáles \& Vázquez, 2009, p. 100).

A sobredotação é um constructo complexo e multidimensional (Almeida et al., 2017). Apesar de a sobredotação não constituir, em si mesma, um inconveniente, têm sido apontadas várias dificuldades em algumas crianças e jovens com estas características, especialmente quando os contextos em que se inserem não se ajustam às suas capacidades, interesses e necessidades específicas (Costa-Lobo, Medeiros, \& Ponte, 2016). O desenvolvimento psicossocial dos sujeitos está sempre em desenvolvimento sendo que as figuras parentais têm uma forte influência no desenvolvimento do sujeito (Berger, 2003; Blair \& Raven, 2012). Todas as crianças e jovens deparam-se com vários problemas ao longo do seu desenvolvimento, porém, as crianças e jovens sobredotados, devido às suas caraterísticas singulares, podem-se deparar com várias questões emocionais e/ou sociais mais vincadas ao longo do seu desenvolvimento (Almeida, Rocha, \& Fonseca, 2016), para que consigam ultrapassar estas questões, é preponderante que detenham um apoio familiar ajustado. De acordo com o estudo de Ogurlu e Yaman em 2013, os pais de crianças e jovens sobredotados expõem diversas dúvidas relacionadas com a educação dos seus filhos, um dos motivos depreende-se com as particularidades das mesmas, necessitando assim de acompanhamento durante os anos escolares, perante estas dúvidas e inquietações, estes pais necessitam de conselhos e informações sobre estratégias parentais (Leana-Tascilar, Ozyaprak, \& Yilmaz, 2016).

Vários autores (e.g. Almeida et al., 2017) defendem que a melhor forma de intervenção com estudantes sobredotados decorre de uma avaliação prévia e de uma avaliação sistemática, supervisionando a implementação das medidas educativas que a cada momento se considerem mais apropriadas, e procurando atender a efeitos primários e a efeitos secundários da própria intervenção. De acordo com Morawska \& Sanders (2009) estas medidas educativas tendem a ter um impacto positivo na resolução de problemas comportamentais dos seus filhos e também tende a ter uma influência nos estilos parentais, como por exemplo, na redução de comportamentos permissivos e melhoria da comunicação com os filhos.

Alguns país de estudantes sobredotados, preocupados com a resposta necessidades educativas destas crianças, organizam a vida familiar tomando esta criança como "centro", o que pode nalgum momento vir a mostrar-se disfuncional (Almeida et al., 2017). Atentando ao estudo de Leana-Tascilar et al., (2016), estes agentes educativos experienciam momentos de desespero e solidão devido às escassas respostas cedidas pelos profissionais, devido a isto, o acompanhamento, durante todo o processo de desenvolvimento dos filhos, é fundamental para assim conseguirem apoiar e reforçar as habilidades dos seus filhos, sendo necessário existirem programas destinados a estes pais, com o objetivo de conceder informações sobre a sobredotação e parentalidade e também com o objetivo de oferecer um espaço onde estes pais sintam a perceção de desespero e solidão diminuída (Almeida, Rocha, \& Fonseca, 2016; Leana-Tascilar et al., 2016).

\section{Bem-Estar Subjetivo}

O Bem-Estar Subjetivo é um conceito recente e bastante complexo que integra uma dimensão cognitiva e uma dimensão afetiva. É considerado como uma dimensão positiva da saúde que abrange outros grandes conceitos como Qualidade de Vida, Afeto Positivo e Afeto Negativo. Devido à sua abrangência é disposto numa posição de interseção de vários domínios da Psicologia, como, a Psicologia Social, a Psicologia da Saúde e também a Psicologia Clínica (Galinha \& Ribeiro, 2005).

De acordo com Diener, Suh \& Oishi em 1997, o BemEstar Subjetivo constitui um campo de estudos que procura compreender as avaliações que os sujeitos produzem das suas próprias vidas.

A história do conceito aponta várias heranças históricas distintas, todas elas relacionadas com a sua convergência de origens teóricas distintas. Identifica-se uma primeira herança nos movimentos sociais inspirados no Iluminismo e no Utilitarismo. $\mathrm{O}$ Iluminismo, no século XVIII, defendia que a propósito da existência da Humanidade era a vida em vez do serviço ao Rei ou a Deus, devido a isso, o desenvolvimento pessoal e a felicidade tornaram-se valores centrais, a sociedade foi vista, pela primeira vez durante esta época, como um meio de proporcionar aos sujeitos a satisfação das suas necessidades. Durante o século XIX, um dos princípios do Utilitarismo era de que a melhor sociedade era a que proporcionava a melhor felicidade para o maior número de sujeitos (Veenhoven, 1996).

Uma segunda herança histórica deste conceito está relacionada com a Segunda Revolução da Saúde, no decorrer da década de 70, onde surgem os conceitos de Promoção de Saúde e de Estilo de Vida.

Lucas, Diener \& Suh, (1996), concluíram que o conceito de Bem-Estar permitiu que fossem construídas medidas que possibilitaram uma avaliação mais precisa dos indicadores da Saúde Mental, através de variáveis como a Satisfação com a Vida, a Felicidade, o Afeto Positivo e o Afeto Negativo, ou seja, o estudo científico do conceito é considerado como o marco de viragem da orientação da Psicologia para a Saúde Mental, assumindo-se como o conceito chave de um campo de investigação emergente designado de Psicologia Positiva (Galinha \& Ribeiro, 2005). 
O conceito de Bem-Estar Subjetivo está associado à psicologia positiva. De acordo com os estudos de Veenhoven (2000) os conceitos de qualidade de vida, Bem-Estar e felicidade são utilizados como sinônimos, porém, Sirgy (2002) inclui os conceitos de satisfação com a vida, afeto positivo, afeto negativo, Bem-Estar Subjetivo, felicidade e a perceção da qualidade de vida como sinônimos.

Na década de 60, o termo Bem-Estar estava associado aos estudos da economia tendo a designação de BemEstar Material que por sua vez era a avaliação feita pelo sujeito ao seu rendimento ou à contribuição dos bens e serviços que o dinheiro poderia comprar para o seu Bem-Estar. Existia uma necessidade de proceder a uma distinção entre o Bem-Estar Material e o Bem-Estar Global, de acordo com Novo (2005), surgiu uma dimensão global de Bem-Estar na vida como um todo, estando outras dimensões valorizadas na vida dos sujeitos.

\section{Metodologia}

\section{Participantes}

A amostra é composta por 30 pais de crianças e jovens que frequentam a Associação Nacional para o Estudo e Intervenção na Sobredotação (doravante designada por ANEIS), na sua delegação da cidade do Porto e a ANEIS Braga, cidade igualmente situada no Norte de Portugal. A totalidade dos participantes concordou voluntariamente em participar no estudo. Do total de pais implicados, $60 \%$ pertencem ao sexo feminino. A idade dos participantes variou de 37 a 56 anos $(M=42,97$ e $D P=4,20)$.

A ANEIS é uma associação sem fins lucrativos, tendo sido criada no ano de 1998 e tem como objetivo prestar apoio a crianças e jovens com caraterísticas de sobredotação como também às suas famílias (Rocha, Fonseca, \& Almeida, 2015). A ANEIS desenvolve programas de enriquecimento, nomeadamente o Programa de Enriquecimento em Domínios da Aptidão, Interesse e Socialização (PEDAIS), que se encontra a ser desenvolvido na ANEIS Porto desde o ano de 2000 tendo como objetivo "promover aprendizagens orientadas para a resolução de problemas retirados da realidade vivencial dos sujeitos em função" (Rocha, Fonseca, \& Almeida, 2015, p.46).

\section{Instrumentos}

Escala de Afetos. A avaliação do Bem-Estar Subjetivo efetuou-se através da escala de afeto positivo e negativo (versão reduzida), adaptada da Positive and Negative Affect Schedule (1988). Originalmente com 60 itens, a escala pretende medir o Afeto Positivo e o Afeto Negativo, definidos como dimensões gerais que descrevem a experiência afetiva dos sujeitos. A adaptação do Positive and Negative Affect Schedule foi realizada por Galinha e Ribeiro em 2005, sendo um instrumento de aplicação individual, composto por 20 itens respondidos em escalas Likert de 5 pontos, variando de muito pouco ou nada (1) a muitíssimo (5), segundo os estados de espirito que o sujeito experienciou num determinado período de tempo (hoje, na última semana, no último mês); o objetivo deste instrumento é avaliar a vertente afetiva do Bem-Estar Subjetivo, sendo constituída por 20 termos descritores da afetividade sentida pelo sujeito, 10 termos referem-se ao afeto negativo e os restantes 10 termos referem-se ao afeto positivo. No presente estudo, o índice de consistência interna da escala, calculado através do coeficiente de Alfa de Cronbach, foi de 0.60.

Programa de Treino Parental. O Programa Parentalidade Positiva é de autoria e é realizado pela ANEIS, delegação do Porto. É um programa de intervenção destinado a pais de crianças e jovens sobredotados tendo como objetivo principal melhorar a qualidade da relação pais-filhos como também a educação prestada (Almeida, Rocha, \& Fonseca, 2016). Este programa é realizado desde o ano de 2013 tendo inicio a cada mês de Janeiro, mantendo uma periocidade mensal e contando com um número de participantes que varia entre 15 a 20 elementos. Este programa tem como base metodológica o Positive Parenting Program (Triple-P) e consiste numa intervenção junto dos pais num formato de grupo (Almeida, Rocha, \& Fonseca, 2016; Sanders, et al., 2014). Durante as sessões do programa são abordados vários temas, como por exemplo, o aconselhamento e o treino de estratégias parentais que ajudam a lidar com questões comportamentais e também emocionais dos seus filhos; e o aumento de competências parentais perante os desafios comportamentais, sociais e emocionais dos seus filhos (Almeida, Rocha, \& Fonseca, 2016).

No inicio de cada ano letivo é realizado um levantamento de necessidades dos pais através de recolha de informações prestadas pelos mesmos tendo como objetivo geral o envolvimento dos mesmos "no envolvimento na educação dos seus filhos, na promoção de competências parentais e formação acerca da sobredotação, tendo a finalidade de fomentar a qualidade de vida e Bem-Estar dos pais bem como da criança" (Almeida, Rocha \& Fonseca, 2016, p.118). A eficácia do Programa Parentalidade Positiva já foi analisada pela instituição contando com valores satisfatórios, tendo no ano letivo de 2014/2015, atingido um nível de elevada satisfação e eficácia da sua aplicação (Almeida, Rocha, \& Fonseca, 2016).

\section{Procedimentos}

A escala foi aplicada individualmente, aplicação com duração aproximada entre 20 e 30 minutos, em seguida à obtenção de autorização dos responsáveis institucionais. Os pais foram esclarecidos da natureza e objetivos do estudo, e após o preenchimento do consentimento informado, foi garantido o anonimato, a confidencialidade, a proteção e a segurança dos dados recolhidos. Recorreu-se a um grupo de controle constituído por pais que não frequentaram o Programa Parentalidade Positiva e cujos filhos frequentam a ANEIS. O grupo de controlo é constituído por pais da ANEIS Braga e o grupo de intervenção é constituído por pais da ANEIS Porto. 


\section{Resultados}

Com o objetivo de se avaliar as diferenças nos níveis de Bem-Estar Subjetivo dos dois grupos, comparam-se os mesmos após a frequência do Programa Parentalidade Positiva. Na tabela 1 apresentam-se estas diferenças.

A análise das diferenças entre o grupo de controlo $(M=32.7, D P=4.0)$ e o grupo de intervenção $(M=29.4$, $D P=7.19)$ permite constatar que não se verificam diferenças estatisticamente significativas $(t=-.096, p=$ $.92)$.

Assim, os afetos positivos parecem não variar em função da frequência do Programa Parentalidade Positiva.

Tabela 1.

Diferenças nos Afetos Positivos em Função da Frequência do Programa Parentalidade Positiva: Teste t-Student

\begin{tabular}{llll}
\hline $\begin{array}{l}\text { Bem-Estar } \\
\text { Subjetivo }\end{array}$ & Grupo & $t$ & $P$ \\
\hline \multicolumn{3}{c}{ Grupo de } \\
Afetos & $\begin{array}{l}\text { Controlo } \\
\text { Positivos }\end{array}$ & $\begin{array}{l}\text { Grupo de } \\
\text { Intervenção }\end{array}$ & \\
\hline
\end{tabular}

Na tabela 2 comparam-se os níveis do afeto negativo em função da frequência do Programa Parentalidade Positiva.

A análise das diferenças entre o grupo de controlo $(M=17.2, D P=5.99)$ e o grupo de intervenção $(M=17.0$, $D P=5.39)$ permite constatar que não se verificam diferenças estatisticamente significativas $(t=-1.5$, $p=.12$ ). Assim, os afetos negativos parecem não variar em função da frequência do Programa Parentalidade Positiva.

Tabela 2.

Diferenças nos Afetos Negativos em Função da Frequência do Programa Parentalidade Positiva: Teste t-Student

\begin{tabular}{llll}
\hline $\begin{array}{l}\text { Bem-Estar } \\
\text { Subjetivo }\end{array}$ & Grupo & $t$ & $p$ \\
\hline & Grupo de & & \\
Afetos & Controlo & -1.56 & .12 \\
Negativos & $\begin{array}{l}\text { Grupo de } \\
\text { Intervenção }\end{array}$ & & \\
& & \\
\hline
\end{tabular}

Através da leitura da tabela 3 , verifica-se que a subescala afetos positivos apresenta uma correlação significativa e positiva com a subescala idade $(r=-.553$, $p=.02)$.

Tabela 3.

Correlação entre o Bem-Estar Subjetivo e a Idade: Correlação de Pearson

\begin{tabular}{ll}
\hline Bem-Estar Subjetivo & Idade \\
\hline Afetos Positivos & $-.553^{* *}$ \\
\hline
\end{tabular}

Os resultados permitem considerar que a afetividade positiva é mais elevada em sujeitos com uma idade mais elevada.

\section{Conclusão}

Este estudo vem corroborar as afirmações de Almeida et al. (2017) apontando a formação dos pais e dos professores, como aliás dos próprios técnicos dos campos da psicologia e da educação especial, como sendo um requisito para se trabalhar de forma mais eficaz na área da sobredotação.

Este estudo suporta a importância das intervenções de formação parental, neste caso no que diz respeito a pais de crianças e jovens sobredotados, com consequências para a qualidade de vida e Bem-Estar nos pais como também nos seus filhos.

Há, com este estudo, reforço da necessidade dos académicos e dos profissionais centrados nas variáveis inerentes à sobredotação convergirem, pois esta área subsiste como uma área de proliferação de conhecimento pouco consistente.

Este estudo faz parte de um projeto mais alargado, projeto no qual está a ser analisada a eficácia do programa aqui sinalizado, Programa Parentalidade Positiva, no que respeita aos seguintes critérios: BemEstar Subjetivo, qualidade de vida e satisfação com a vida.

\section{Referências}

Almeida, L. S., Costa-Lobo, C., Almeida, A. I. S., Rocha, R. S., \& Piske, F. H. R. (2017). Processos cognitivos e de aprendizagem em crianças sobredotadas: atenção dos pais e professores. In F. H. R. Piske, C. L. B. Vestena, J. M. M., A. A. de O. M. Barby, T. Stoltz, S. Bahia, \& S. P de Freitas (Eds.). Processos afetivos e cognitivos de superdotados e talentosos: Curitiba : Editora Prismas, 15-39.

Almeida, I. S., Rocha, A., \& Fonseca, H. (2016). Programa Parentalidade Positiva: Programa de Intervenção Parental de Crianças e Jovens Sobredotados. Sobredotação, 15 (1), 113-131.

Baptista, T. (2014). Apoio Social Percebido: Necessidades e Satisfação numa Amostra de Famílias com Menores em Risco Psicossocial. Dissertação de Mestrado, Universidade do Algarve, Portugal.

Berger, K. S. (2003). O desenvolvimento da pessoa: da infância à terceira idade. Rio de Janeiro: LTC.

Blair, C. \& Raven, C. (2012). Child Development in the Context of Adversity: Experiential Canalization of Brain and Behavior. American Psychologist, 67(4), 309-318.

Corvo, J. (2015). Parentalidade Positiva e a prevenção de condutas de risco em adolescentes: o papel do Criminólogo. Dissertação de Mestrado, Universidade Fernando Pessoa Faculdade de Ciências Humanas e Sociais, Portugal.

Costa-Lobo, C., Medeiros, A. M., \& Ponte, F. E. (2016). Sobredotação e autoeficácia percebida: uma revisão teórica. Revista AMAzônica, 9(2), 149-175.

Diener, E., Suh, E., \& Oishi, S. (1997). Recent Findings on Subjective Well-Being. Indian Journal of Clinical Psychology, 1-24 
Galinha, I. \& Ribeiro, J. (2005). História e evolução do conceito de Bem-Estar Subjetivo. Psicologia, saúde e doenças, 6 (2), 203-214

González, M. \& Vázquez, C. (2009). Orientación Educativa para la Vida Familiar como Medida de Apoyo para ele Desempeño de la Parentalidad Positiva. Intervención Psicosocial, 18(2), 97-112.

Leana-Tascilar, M. Z., Ozyaprak, M., \& Yilmaz, O. (2016). An Online Training Program for Gifted Children's Parents in Turkey. Eurasian Journal of Educational Research, 65, 147-164.

Lopes, M., S., Catarino, H., \& Dixe, M. (2010). Parentalidade Positiva e Enfermagem: Revisão Sistemática da literatura. Revista Enfermagem Referência, 3 (1), 109-118.

Lucas, R., E., Diener, E., \& Suh, E. (1996). Discriminant Validity of Well- Being Measures. Journal of Personality and Social Psychology, 71 (3), 616-628.

Martins, C. (2013). Transição no exercício da parentalidade durante o primeiro ano de vida da criança: uma teoria explicativa de enfermagem. Dissertação de Doutoramento, Escola Superior de Enfermagem de Lisboa, Portugal.

Morawska, A., \& Sanders, M. (2009). An evaluation of a behavioural parenting intervention for parents of gifted children. Behaviour Research and Therapy, 47, 463-470.

Novo, R., F. (2005). Bem-Estar e Psicologia: Conceitos e Propostas de Avaliação. Revista Iberoamericana de Diagnóstico y Evaluación Psicológica, 2 (20), 183203.

Ogurlu, Ü., \& Yaman, Y. (2013). Guidance Needs of Gifted and Talented Children's Parents. Turkish Journal of Giftedness and Education, 3(2), 81-94.

Patrício, S. (2011). Promoção da Parentalidade Positiva. Dissertação de Mestrado, Universidade Católica Portuguesa, Portugal.

Rocha, A., Fonseca, H., \& Almeida, A. (2015). Programa de Enriquecimento em Domínios da Aptidão, Interesse e Socialização (PEDAIS): Uma proposta de enriquecimento para alunos com altas capacidades. Talincrea, 2(1), 35-56.

Sanders, M. R., Kirby, J. N., Tellegen, C. L., \& Day, J. J. (2014). The Triple P-Positive Parenting Program- A systematic review and meta-analysis of a multi-level system of parenting support. Clinical Psychology Review, 34(4), 337-357.

Sirgy, M. (2002). The Psychology of Quality of Life. Estados Unidos: Springer Science \& Business Media

Veenhoven, R. (1996). The Study of Life Satisfaction. Quality, 11-48.

Veenhoven, R. (2000). The four qualities of life: Ordering concepts and measures of the good life. Journal of Happiness Studies, 1, 1-39. 